tion * of the matrix $A$, in order to express the higher powers of $A$. This leads to tedious work unless the characteristic equation reduces to one in two terms only; for instance, types V and VI (in Slocum's list of three-parameter groups) lead to the equations for $A$

$$
A^{2}+a_{3} A=0, \quad A^{2}=0
$$

respectively. This method has been used by Dr. H. F. Baker in a recent paper on the calculation of the finite equations of a group from its structural constants (Proceedings of the London Mathematical Society, volume 34 (1902), page 91); but Baker's work relates solely to the determination of the matrix which is denoted by $B$ in my notation, $i$. e., the matrix reciprocal to $f(A)$.

I have not actually applied the last method to any of the harder cases ; indeed, I have only used it for the two cases just mentioned, when it gives

$$
\begin{array}{ll}
\text { Type V. } & f(A)=E+\left(\frac{1}{a_{3}}-\frac{1}{e^{a_{3}}-1}\right) A . \\
\text { Type VI. } & f(A)=E+\frac{1}{2} A .
\end{array}
$$

It may, however, prove useful as an alternative means of verification.

Queen's College, GalWAY, February 25, 1902.

\title{
ON THE PARABOLAS (OR PARABOLOIDS) THROUGH THE POINTS COMMON TO TWO GIVEN CONICS (OR QUADRICS).
}

BY PROFESSOR T. J. I'A. BROMWIGH.

(Read before the American Mathematical Society, April 26, 1902.)

In the December issue of the Bulletin (page 122, December, 1901) Huntington and Whittemore have called attention to the features of conics which touch the line infinity

* This is obtained as follows: Let $\phi(t)$ be the quotient of the determinant $|t E-A|$ by the highest common factor of all its first minors. Then the equation is

$$
\phi(A)=0 \text {. }
$$

See Frobenius, Crelle, vol. 84 ; and other references given by the present author in a review, Bulletis, vol. 7 (1900), p. 308. 
at one of the circular points. Apparently they were led to the discussion of these conics by considering the disappearance of parabolas from the family $S+\lambda S^{\prime}=0$, when $S=0$, $S^{\prime}=0$ are rectangular hyperbolas. This recalls to my miud a neat result which I have used in class-work, but have never seen in print, namely :

The two parabolas contained in the family $S+\lambda S^{\prime}=0$ are real, if either of the two conics $S=0, S^{\prime}=0$ is an ellipse; this condition is sufficient but not necessary.

This is at once obvious if we write in cartesians

$$
S=a x^{2}+2 h x y+b y^{2}+\cdots, \quad S^{\prime}=a^{\prime} x^{2}+2 h^{\prime} x y+b^{\prime} y^{2}+\cdots,
$$

for then the condition for a parabola is

$$
\left|\begin{array}{cc}
a-\lambda a^{\prime}, & h-\lambda h^{\prime} \\
h-\lambda h^{\prime}, & b-\lambda b^{\prime}
\end{array}\right|=0,
$$

which will have real roots if either $a b-h^{2}>0$, or $a^{\prime} b^{\prime}-h^{\prime 2}>0$ (by Weierstrass's general theorem, Monatsberichte d. k. Akad. z. Berlin, 1858; Werke, volume 1, page $233, \S 3)$.

Suppose now that $S$ and $S^{\prime}$ are hyperbolas; then a short piece of arithmetic will prove that the necessary condition that the values of $\lambda$ may be real is that the two pairs of lines

$$
a x^{2}+2 h x y+b y^{2}=0, \quad a^{\prime} x^{2}+2 h^{\prime} x y+b^{\prime} y^{2}=0
$$

do not interlace (cf. Chrystal's Algebra, volume 1, p. 464). 'These pairs of lines are, of course, parallel to the asymptotes of $S$ and $S^{\prime}$, and so, if $S, S^{\prime}$ are rectangular hyperbolas, it is clear that the parabolas of the system must be imaginary.

Proceeding next to the possibility of coincident parabolas in the family $S+\lambda S^{\prime}$, we have first, when $S$ or $S^{\prime}$ is an ellipse, that the equation for $\lambda$ has equal roots only if

$$
\frac{a}{a^{\prime}}=\frac{h}{h^{\prime}}=\frac{b}{b^{\prime}}=\lambda
$$

as is seen by a second theorem due to Weierstrass (l. c., $\$ 4)$. In this case, each parabola degenerates to a line and the line at infinity.

Second, if $S, S^{\prime}$ are both hyperbolas, we may have the same result as just given. Or, we may have one asymptote of $S$ parallel to one of $S^{\prime}$, and then the coincident parabolas of $S+\lambda S^{\prime}$ are not necessarily degenerate; the axis of the 
resulting parabola is parallel to the two parallel asymptotes.

An extension to space of three dimensions is easy; thus the analogue to the first theorem gives

The three paraboloids contained in the family $S+\lambda S^{\prime}=0$ are all real, if either of the quadrics $S, S^{\prime}$ is an ellipsoid. So too, we find :

If the two quadrics $S, S^{\prime}$ are hyperboloids, two of the paraboloids will be imaginary if (and only if) two cones with a common vertex, parallel to their asymptotic cones, intersect in two real generators.

There are five possibilities when two (or three) of the paraboloids coincide; without enumerating them all, it may be noted that when $S$ or $S^{\prime}$ is an ellipsoid, the coincidence implies degeneration of the paraboloids. All the other cases may be obtained by suitable interpretations of Weierstrass's algebra ("Zur Theorie der bilinearen und quadratischen Formen," Monatsberichte d. k. Akad. z. Berlin, 1868 ; Werke, volume 2, page 19).

Slightly digressing from the line of thought just indicated, and reverting to Huntington and Whittemore's paper, I note that their result, that the eccentricity is wholly indeterminate (l. c., page 123), suffices to specify the conics considered by them. For, in orthogonal cartesians, the eccentricity is determined by the ratio $(a+b)^{2} /\left(a b-h^{2}\right)$, which is only indeterminate if

i. e., if

$$
\begin{gathered}
a b-h^{2}=0, \quad a+b=0, \\
b=-a, \quad h= \pm i a,
\end{gathered}
$$

and then the conic reduces to

$$
a(x \pm i y)^{2}+\text { linear terms }=0 .
$$

QueEN's CoLlege, GaLWAY, February $22,1902$.

\section{A SECOND DEFINITION OF A GROUP.}

BY DR. E. V. HUNTINGTON.

(Read before the American Mathematical Society, April 26, 1902.)

THE following note contains a definition of a group expressed in four independent postulates, suggested by the definition given in W. Burnside's Theory of Groups of Finite Order (1897). The definition presented by the writer at the February meeting contained three independent 